\title{
Amplitude-Probability Distribution of Atmospheric Radio Noise
}

\author{
Petr Beckmann ${ }^{1}$ \\ Contribution From Department of Electrical Engineering, University of Colorado, Boulder, Colo.
}

(Received November 20, 1963)

\begin{abstract}
Atmospherics are assumed to be spike shaped with exponential upsurge and decay; their occurrence in time is Poisson-distributed. The distribution of the peak value is shown to be lognormal. Under these conditions, the amplitude distribution of atmospheric noise is derived; it may be well approximated by the distribution of the sum of a Rayleigh vector and a vector with lognormally distributed amplitude. The resulting distribution is uniquely determined by three physically meaningful parameters and is in very good agreement with measured distributions.
\end{abstract}

\section{Introduction}

The probability distribution of the amplitude of atmospheric radio noise is an important basic factor for the design of radio systems; it may also be used as an effective instrument for geophysical research of atmospheric discharges by which this noise is caused. The statistical distribution of the amplitude of atmospheric radio noise is therefore being measured in observatories all over the world; so far it has, however, not been possible to give a thorough and practically useful theoretical explanation of this distribution.

It has been found empirically that the distribution

$$
P(E>R)
$$

where $E$ is the instantaneous value of the envelope of the HF atmospheric noise and $R$ is a selected level of voltage or field strength at the receiving point, approaches a Rayleigh distribution for small amplitudes (high probabilities). This is evidently caused by the interference of many mutually overlapping small atmospheries whose phase is uniformly distributed over a basic phase cycle, for these atmospherics are emitted by independent sources at distances of many wavelengths from the receiver and from each other. For large amplitudes (low probabilities) the distribution deviates considerably from a Rayleigh distribution and may be well represented by a lognormal distribution [Horner and Harwood, 1956; Foldès, 1960]. This part of the distribution curve is evidently due to relatively rare, strong atmospheries that do not overlap in time.

It has also been found empirically [Crichlow et al., 1960] that when the experimentally measured distribution (1.1) is mapped on Rayleigh paper (cf, appendix), the lower portion of the curve ( $\operatorname{small} R$ ) is a straight line with slope corresponding to the Rayleigh distribution and the upper portion of the curve (large $R$, small $P$ ) may be adequately represented by a straight line of larger slope than that corresponding to a Rayleigh distribution. As shown in the appendix (and fig. 1), a lognormal distribution will map on Rayleigh paper in this way. Between the two straight lines there is a transition that has been approximated by Crichlow et al. [1960] as a circular arc.

The individual atmospherics that protrude from the permanent noise level are spike shaped and decay rapidly, exceeding the permanent noise level only for some milliseconds [Horner and Harwood, 1956]; this order of time is in agreement with the characteristics of lightning discharges [Watt and Maxwell, 1957]. The decay is evidently exponential and has

1 On leave of absence from Institute of Radio Engineering and Electronics, Czechoslovak Academy of Sciences, Prague 8, Czechoslovakia. 


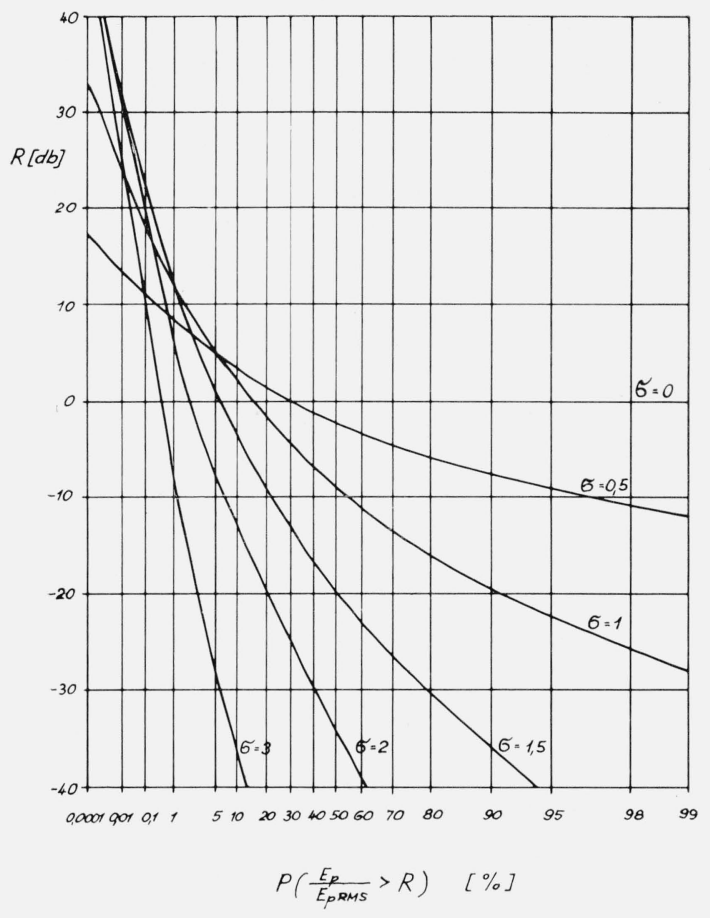

Figure 1. Lognormal distributions plotted on Rayleigh paper.

a small time constant. The buildup is also exponential with a still shorter tirne constant.

The purpose of this paper is to derive the theoretical probability distribution (1.1). In section 2 we shall first derive the probability distribution of the peak values of the individual atmospherics. In section 3 we derive some general probabilistic relations, in particular the distribution of the time interval separating a peak from the $k$ th following or the $k$ th preceding peak assuming the number of peaks in a given time interval to be Poisson distributed. In section 4 we derive the amplitude distribution of the decaying $k$ th preceding atmospheric and the rising $k$ th future atmospheric; the time constants of rise and decay may be chosen individually and arbitrarily, so that we are working with a very general spike-shaped atmospheric. In section 5 the rms value of an individual atmospheric and of the entire atmospheric noise is derived and the required distribution is then found by summing over all $k$; the resulting distribution is the sum of a Rayleigh vector and a vector with lognormally distributed amplitude. In section 6 this distribution is compared with the experimentally measured distribution; in spite of some coarse approximations, the agreement is surprisingly good. As in the case of the empirically graphical method of Crichlow et al. [1960], the resulting distribution is uniquely given by three parameters; as one of these depends exclusively on the properties of atmospheric discharges and one predominantly on propagation conditions (the third drops out in normalizing to the rms value), the theoretical distribution derived in this paper should prove useful for geophysical research.

\section{Distribution of the Peak Value of Atmospherics}

The peak value $E_{p}$ of an atmospheric is determined by the peak power $P_{0}$ of the atmospheric discharge emitting it, the distance of the discharge from the receiving point, and the attenuation $\Delta$ in the propagation of the atmospheric (all at the considered frequency); therefore

$$
E_{p}=\frac{K \sqrt{P_{0}}}{d} \exp \left(-\sum_{j=1}^{n} \delta_{j} d_{j}\right)
$$

where $K$ is a constant determined by the system of units employed; the attenuation $\Delta$ is given by the sum of attenuations on the individual sections $d_{j}$ of the propagation path (whose total 
length is $d) ; \delta_{j}$ is the attenuation constant for the $j$ th section. We may thus write (2.1) as

$$
E_{p}=e^{\Delta}=\exp \left(-\sum_{j=1}^{n} \delta_{j} d_{j}+\frac{1}{2} \ln P_{0}-\ln \sum_{j=1}^{n} d_{j}+\ln K\right)
$$

which defines $\Delta$.

The first three terms of the exponent are random; we may assume that the fluctuations (the variance) of $P_{0}$ and $d$ will, on taking the logarithm of these quantities, be so much diminished that they will be small compared with the fluctuations (the variance) of the sum of attenuations as given by the first term of the exponent, which will thus predominate in determining the distribution of $\Delta$. We further assume that the total propagation path $d$ can be split up into a large number of sections $d_{j}$ on which the attenuations $\delta_{j} d_{j}$ may be considered independent with no section making a predominant contribution to the sum of attenuations. This assumption seems reasonable since most atmospherics are propagated from long distances via the ionosphere. Then $\Delta$ will be distributed normally, i.e., its probability density will be

$$
p(\Delta)=\frac{1}{\sigma \sqrt{2 \pi}} \exp \left[-\frac{(\Delta-\mu)^{2}}{2 \sigma^{2}}\right]
$$

where $\mu$ is the mean value of $\Delta$ and $\sigma^{2}$ its variance.

From (2.2) we have

$$
\Delta=\ln E_{p}
$$

which together with (2.3) yields the required distribution of $E_{p}$ :

$$
\lambda\left(E_{p}\right)=\frac{1}{\sigma E_{p} \sqrt{2 \pi}} \exp \left[-\frac{\left(\ln E_{p}-\mu\right)^{2}}{2 \sigma^{2}}\right]
$$

and hence

$$
P\left(E_{p}>R\right)=\Lambda(R)=\int_{R}^{\infty} \lambda\left(E_{p}\right) d E_{p}=\frac{1}{2}\left[1-\operatorname{erf}\left(\frac{\ln R-\mu}{\sigma \sqrt{2}}\right)\right]
$$

The distribution (2.5) or (2.6) is the logarithmic-normal distribution, which has been found experimentally to apply to strong atmospherics [Horner and Harwood, 1956 ; Foldés, 1960] but was not explained theoretically. The mean and mean square values of $E_{p}$ are from (2.5)

$$
\begin{aligned}
\left\langle E_{p}\right\rangle & =\exp \left(\mu+\frac{\sigma^{2}}{2}\right) \\
\left\langle E_{p}{ }^{2}\right\rangle & =\exp 2\left(\mu+\sigma^{2}\right) .
\end{aligned}
$$

From (2.8) and (2.6) we then have

$$
P\left(\frac{E_{p}}{E_{p \mathrm{rms}}}>R\right)=\frac{1}{2}\left[1-\operatorname{erf}\left(\frac{\ln R+\sigma^{2}}{\sigma \sqrt{2}}\right)\right]
$$

If we wish to express $R$ in decibels, we obtain after elementary manipulations

$$
P\left(\frac{E_{p}}{E_{p \mathrm{rms}}}>R_{\mathrm{db}}\right)=\frac{1}{2}\left[1-\operatorname{erf}\left(\frac{R_{\mathrm{db}}+A \sigma^{2}}{A \sigma \sqrt{2}}\right)\right]
$$

where

$$
A=20 \log _{10} e=8.686 \text {. }
$$

Curves of (2.10) for various values of $\sigma$ are plotted on Rayleigh paper in figure 1. 


\section{Probabilistic Relations}

In order not to interrupt our development later, we now derive two general relations that we shall need.

Since atmospherics are emitted by mutually independent sources and the reception of an atmospheric is equally probable at any time, their number in a given time interval will be Poisson distributed, i.e., the probability of receiving $n$ atmospherics in a time interval of length $\tau$ is

$$
P(n ; \tau)=\frac{(N \tau)^{n}}{n !} e^{-N \tau},
$$

where $N$ is the mean number of atmospherics received per unit time.

To find the probability density of the time interval $T_{k}$ separating a given peak from the $k$ th preceding peak, i.e.,

$$
p_{k}\left(t_{k}\right)=\operatorname{Pr}\left(t_{k} \leq T_{k} \leq t_{k}+d t_{k}\right)
$$

we note that this probability equals that of receiving $k-1$ atmospherics during the time $t_{k}$ and one atmospheric during $d t_{k}$; since the two events are independent, we have

$$
p_{k}\left(t_{k}\right)=P\left(k-1 ; t_{k}\right) P\left(1 ; d t_{k}\right)
$$

or substituting from (3.1),

$$
p_{k}\left(t_{k}\right)=\frac{N^{k} t_{k}^{k-1}}{(k-1) !} e^{-N t_{k}}
$$

which is the first relation we shall require.

The second concerns a random signal (vector) with independent amplitude and phase

$$
\hat{E}=E e^{i \phi}
$$

where we have suppressed the time factor $\exp (i \omega t)$. Let the phase be distributed uniformly over an interval of length $2 \pi$, or more generally let the probability density of the phase, $p(\phi)$, be such that for all $\phi$ in the interval $0<\phi<2 \pi$

$$
\sum_{k=-\infty}^{\infty} p(\phi+2 k \pi)=\frac{1}{2 \pi}+\epsilon(\phi) ; \quad|\epsilon(\phi)|<<\frac{1}{2 \pi}
$$

so that $p(\phi)$ is equivalent to a uniform distribution in the interval $(0,2 \pi)$. A vector of the kind (3.5), whose phase distribution satisfies (3.6), is met so often in wave propagation that it deserves a name; because of its Uniformly Distributed Phase we shall call it a UDP vector. (A Rayleigh vector is the special case of a UDP vector with Rayleigh-distributed amplitude.) The sum of any number of UDP vectors

$$
\hat{U}=U e^{i \theta}=\sum_{j=1}^{n} \hat{E}_{j}=\sum_{j=1}^{n} E_{j} e^{i \phi_{j}}
$$

is obviously again a UDP vector. Consider now the mean square value of a UDP-vector sum

$$
\left\langle U^{2}\right\rangle=\left\langle\hat{U} \hat{U}^{*}\right\rangle=\left\langle\sum_{j=1}^{n} E_{j} e^{i \phi_{j}} \sum_{k=1}^{n} E_{k} e^{-i \phi_{k}}\right\rangle=\sum_{j=1}^{n}\left\langle E_{j}^{2}\right\rangle+\sum_{j \neq k}\left\langle E_{j} E_{k}\right\rangle\left\langle e^{i\left(\phi_{j}-\phi_{k}\right)}\right\rangle \cdot
$$

But for $j \neq k$,

$$
\left\langle e^{i\left(\phi_{j}-\phi_{k}\right)}\right\rangle=\frac{1}{4 \pi^{2}} \int_{0}^{2 \pi} \int_{0}^{2 \pi} e^{i\left(\phi_{j}-\phi_{k}\right)} d \phi_{j} d \phi_{k}=0 .
$$

Substituting (3.7) and (3.9) in (3.8) we find

$$
\left\langle\left|\sum_{j=1}^{n} \hat{E}_{j}\right|^{2}\right\rangle=\sum_{j=1}^{n}\left\langle E_{j}^{2}\right\rangle
$$


regardless of the distributions (possibly all different) of the amplitudes $E_{j}$. Thus in the interference of waves that may be represented by UDP vectors, the mean total power equals the sum of mean powers of each wave regardless of the amplitude distributions of the latter.

The phase of an atmospheric is, among other factors, determined by the distance of the atmospheric discharge from the receiver. Since this distance is random and varies over a range much larger than one wavelength, (3.6) is satisfied and any atmospheric may be represented by a UDP vector.

\section{Amplitude Distribution of the Signal Due to the $k$ th Decaying Atmospheric}

We assume the shape of the envelope (amplitude) of an individual atmospheric attaining its peak value $E_{p}$ at time $t_{0}$ of the form

$$
u_{0}(t)=\left\{\begin{array}{l}
\left(E_{p}\right)_{0} \exp \left(-\frac{t-t_{0}}{a}\right) \text { for } t>t_{0} \\
\left(E_{p}\right)_{0} \exp \left(\frac{t-t_{0}}{b}\right) \text { for } t<t_{0}
\end{array}\right.
$$

i.e., in the shape of a spike as shown in figure 2a with arbitrary time constants of decay and upsurge $a$ and $b$. This shape evidently corresponds to the real shape of atmospherics better than rectangular impulses (cf, fast film recordings by Horner and Harwood [1956]).

However, in addition to this atmospheric $u_{0}\left(t_{0}\right)=\left(E_{p}\right)_{0}$ there will be present also an infinite number of decaying atmospherics $u_{1}, u_{2}, \ldots . u_{k},$. . ., that have attained their peak value $\left(E_{p}\right)_{k}$ in the past, $t_{k}$ time units ago (fig. $2 \mathrm{~b}$ ); the distribution of $t_{k}$ is given by (3.4). The
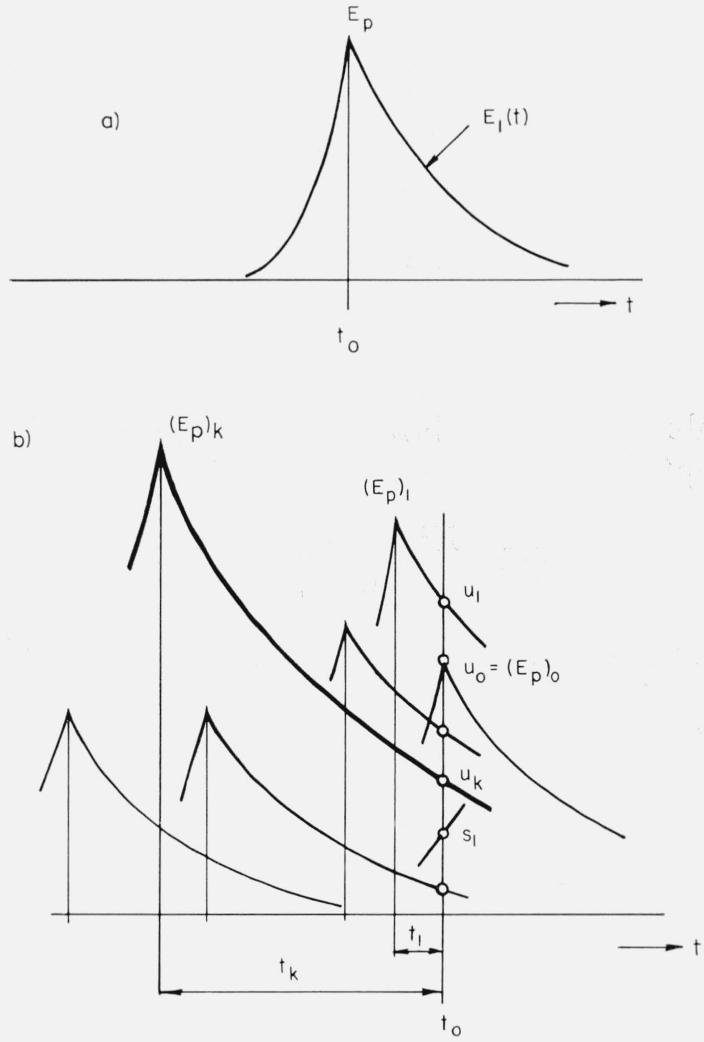

Figure 2. Random sequence of atmospherics. 
amplitude of the $k$ th (past) atmospheric at time $t_{0}$ is therefore

where

$$
u_{k}=\left(E_{p}\right)_{k} \exp \left(-\frac{t_{k}}{a}\right)=\frac{A}{B}
$$

$$
A=\left(E_{p}\right)_{k}, \quad B=\exp \left(\frac{t_{k}}{a}\right)
$$

are independent random variables. The distribution of $A$ is $\lambda(A)$ as given by (2.5). The distribution of $B$ is found from (3.4) by transforming in accordance with (4.3):

$$
w(B)=\frac{N^{k} a^{k}(\ln B)^{k-1}}{(k-1) ! B^{a N+1}} \quad(1<B<\infty) .
$$

The distribution of $u$ is now found from (4.2) as the distribution of the ratio of two independent random variables [Gnedenko, 1954]:

$$
p\left(u_{k}\right)=\int_{1}^{\infty} z \lambda(u z) w(z) d z .
$$

Substituting $\ln z=x$ we find

$$
p\left(u_{k}\right)=\frac{N^{k} a^{k}}{u_{k}(k-1) ! \sigma \sqrt{2 \pi}} \int_{0}^{\infty} x^{k-1} \exp \left[-N a x-\frac{\left(\ln u_{k}+x-\mu\right)^{2}}{2 \sigma^{2}}\right] d x .
$$

The mean square value of $u_{k}$ is

$$
\left\langle u_{k}^{2}\right\rangle=\int_{0}^{\infty} u_{k}^{2} p\left(u_{k}\right) d u_{k}
$$

and may be found from (4.6) by interchanging the order of integration and the substitutions $\ln u=y,(N a+2) x=t$ :

$$
\left\langle u_{k}^{2}\right\rangle=\frac{N^{k} a^{k} \exp \left[2\left(\sigma^{2}+\mu\right)\right]}{(N a+2)^{k}(k-1) !} \int_{0}^{\infty} t^{k-1} e^{-t} d t .
$$

The integral equals $\Gamma(k)=(k-1)$ !, so that we obtain ${ }^{2}$

$$
u_{k}^{2}=\frac{N^{k} a^{k} \exp \left[2\left(\sigma^{2}+\mu\right)\right]}{(N a+2)^{k}} .
$$

Besides the decaying atmospheries $u_{k}$, we have to consider also the upsurging atmospherics $s_{k}$ attaining their peak value at a time $t_{0}+t_{k}$. For the upsurging atmospherics the calculation is of course the same except that the time constant $a$ must be replaced by $b$ so that

$$
\left\langle s_{k}^{2}\right\rangle=\frac{N^{k} b^{k} \exp \left[2\left(\sigma^{2}+\mu\right)\right]}{(N b+2)^{k}} .
$$

\section{Amplitude Distribution of Atmospheric Noise}

The total signal $\hat{U}$ at time $t_{0}$ is

$$
\hat{U}=\hat{u}_{0}+\sum_{k=1}^{\infty} \hat{u}_{k}+\sum_{k=1}^{\infty} \hat{s}_{k}
$$

2 More generally, we find by the above method

$$
\left\langle u_{k}^{m}\right\rangle=\left(\frac{N a}{N a+m}\right)^{k} \exp \left(\frac{1}{2} m^{2} \sigma^{2}+m \mu\right)
$$

of which (4.9) is a special case for $m=2$. 
where the circumfiex accents again denote UDP vectors. Applying (3.10), we therefore find from (2.8), (4.9), and (4.10)

$$
\left\langle U^{2}\right\rangle=\exp \left[2\left(\sigma^{2}+\mu\right)\right]\left\{1+\sum_{k=1}^{\infty}\left(\frac{N a}{N a+2}\right)^{k}+\sum_{k=1}^{\infty}\left(\frac{N b}{N b+2}\right)^{k}\right\} .
$$

Both series are geometric with sum $N a / 2$ and $N b / 2$ respectively. Introducing the average time constant of upsurge and decay

$$
c=\frac{a+b}{2}
$$

we then have

$$
\left\langle U^{2}\right\rangle=(1+N c) \exp \left[2\left(\sigma^{2}+\mu\right)\right] .
$$

This is the mean square value of the field at a time $t_{0}$, the time of arrival (peak) of an atmospheric. The probability distribution of the amplitude at that time is strongly dependent on the value of $N c$. It is easily shown from (5.1) and (5.4) that for $N c>>1$, which corresponds to the atmospherics overlapping in time at all but the highest levels, $U$ is approximately Rayleigh distributed; this contradicts the measured distribution.

On the other hand, if $N c<<1$, the two series in (5.2) will be much smaller than unity; in this case the lognormally distributed quantity $u_{0}$ will predominate and the overlapping decaying or upsurging atmospherics $u_{k}$ and $s_{k}$ will affect the resulting distribution only when $u_{0}$ (and hence $U$ ) is small. This is in agreement with the measured distribution and we therefore henceforth assume $N c<<1$. Also, we may safely assume $b<<a$, i.e., that the time constant of the upsurge is much smaller than that of the decay (cf, recordings by Horner and Harwood [1956]). We thus assume

$$
N c \approx \frac{N a}{2}<<1 .
$$

It follows from (5.1) that $\hat{U}$ is a UDP vector composed of infinitely many UDP vectors, of which one $\left(\hat{u}_{0}\right)$ has a mean square comparable to the mean square of the sum, whereas the mean squares of all the others are much smaller and these remaining vector terms will affect the distribution of $E$ only for small amplitudes of $u_{0}$ and hence of $U$. The sum of these remaining vectors is that of a rapidly converging geometric series and thus the conditions for it to be a pure Rayleigh vector are not strictly satisfied [Beckmann, 1962a], though its distribution curve will asymptotically approach a Rayleigh distribution for small amplitudes and thus this sum may well be approximated by a Rayleigh vector. Thus $U$ as in (5.1) will equal the sum of a lognormally distributed vector and a Rayleigh vector. The distribution of the amplitude of the sum is then given by [Beckmann, 1962b]

$$
w(U)=\frac{2 U}{M} e^{-U^{2} / M} \int_{0}^{\infty} \lambda\left(u_{0}\right) e^{-u_{0}^{2} / M} I_{0}\left(2 u_{0} U / M\right) d u_{0}
$$

where $M$ is the mean square value of the Rayleigh vector and $I_{0}$ is the modified Bessel function. It can be shown [Beckmann, 1962b] that for small values of $U$, (5.6) will approach a Rayleigh distribution, whilst for large values of $U$ it will approach a lognormal distribution.

However, it should be pointed out that (5.6) is the distribution of the amplitude at time $t_{0}$, i.e., at the time of arrival (peak) of an atmospheric. For any arbitrary time $t$ (between two successive peaks) the amplitude is

$$
E=U e^{-t / a}
$$

(where the upsurge has been neglected since $b<<a$ ) and although $U$ is random, once it has assumed a certain value, $E$ is governed by the deterministic exponential law (5.7) between two consecutive atmospherics. Thus $\hat{E}$ at any time is also the sum of a lognormal and a Rayleigh vector (both are UDP vectors). If the time interval separating two successive 
atmospherics is $\tau$, then from (5.7)

or using (5.5),

$$
\left\langle E^{2} \mid \tau\right\rangle=\left\langle U^{2}\right\rangle \frac{1}{\tau} \int_{0}^{\tau} e^{-2 t / a} d t=\frac{a}{2 \tau}\left\langle U^{2}\right\rangle\left(1-e^{-2 \tau / a}\right)
$$

$$
\left\langle E^{2} \mid \tau\right\rangle=\left\langle U^{2}\right\rangle \frac{c}{\tau}\left(1-e^{-\tau / c}\right) .
$$

Now $\tau$ is itself a random quantity with probability density $N e^{-N_{\tau}}$, i.e., (3.4) for $k=1$; applying the Theorem of Total Probability to (5.9) we therefore have

$$
\left\langle E^{2}\right\rangle=\left\langle U^{2}\right\rangle N c \int_{0}^{\infty} \tau^{-1}\left(1-e^{-\tau / c}\right) e^{-N \tau} d \tau=\left\langle U^{2}\right\rangle N c \ln \frac{1+N c}{N c}
$$

or from (5.4) and (5.5)

$$
\left\langle E^{2}\right\rangle=(1+N c) N c e^{2\left(\sigma^{2}+\mu\right)} \ln \frac{1+N c}{N c} \approx N c e^{2\left(\sigma^{2}+\mu\right)} \ln (1 / N c) .
$$

Similarly, the mean square value of $E_{i}=u_{i} \exp (-t / a)$ is

in particular,

$$
\left\langle E_{i}^{2}\right\rangle=\left\langle u_{i}^{2}\right\rangle N c \ln (1 / N c),
$$

$$
\left\langle E_{0}^{2}\right\rangle=N c e^{2\left(\sigma^{2}+\mu\right)} \ln (1 / N c) .
$$

It follows from (5.11) and (5.12) that the predominant term in $\hat{E}$ is $\hat{E}_{0}$, whilst the sum of all remaining terms

$$
\hat{Z}=\sum_{i=1}^{\infty} \hat{Z}_{i}
$$

which is Rayleigh distributed, has a mean square value of only

$$
\left\langle Z^{2}\right\rangle=\left\langle E^{2}\right\rangle-\left\langle E_{0}^{2}\right\rangle=N c\left\langle E_{0}^{2}\right\rangle=N^{2} c^{2} e^{2\left(\sigma^{2}+\mu\right)} \ln (1 / N c) .
$$

To find the distribution of $E_{0}$ we use $E_{0}=U_{0} e^{-t / a}$ where $U_{0}$ is lognormal, $t$ is uniform from 0 to $\tau$ and $\tau$ is distributed according to (3.4) for $k=1$; hence

$$
p_{0}\left(E_{0}\right)=N \int_{0}^{\infty} \frac{e^{-N \tau}}{\tau} \int_{0}^{\tau} \lambda\left(E_{0} e^{t / a}\right) e^{t / a} d t d \tau=\frac{N a}{2 E_{0}} \int_{0}^{\infty} \frac{e^{-N \tau}}{\tau}\left[\operatorname{erf}\left(\frac{\ln E_{0}+\tau / a-\mu}{\sigma \sqrt{2}}\right)-\operatorname{erf}\left(\frac{\ln E_{0}-\mu}{\sigma \sqrt{2}}\right)\right] d \tau .
$$

This integral cannot be evaluated in a closed form, but approximate calculations show that the distribution of $E_{0}$ remains roughly lognormal, especially for large values of $E_{0}$ (where the exponential decay distorts the distribution of $U_{0}$ less than for low values attained after a longer time $\tau$ ). Thus if we replace the random variable $\tau$ by its mean value $\langle\tau\rangle=1 / N$ and use (5.5), we find

$$
p_{0}\left(E_{0}\right) \approx \frac{N c}{E_{0}} \operatorname{erfc}\left(\frac{\ln E_{0}-\mu}{\sigma \sqrt{2}}\right)
$$

which for large values of the argument (i.e., large $E_{0}$ ) will asymptotically tend to

$$
p_{0}\left(E_{0}\right) \approx \frac{2 N c \sigma^{2}}{\ln E_{0}-\mu} \cdot \frac{1}{\sigma E_{0} \sqrt{2 \pi}} \exp \left[-\frac{\left(\ln E_{0}-\mu\right)^{2}}{2 \sigma^{2}}\right]
$$

thus retaining the character of a lognormal distribution. Alternatively, one may expand the square bracket in (5.14) in powers of $\tau$; this again yields a lognormal distribution that may be factored out of the integral. Other approximations of (5.14) again retain a lognormal character.

We therefore approximate (5.14) by a lognormal distribution with mean square (5.13). 
Since (5.13) may be written as

$$
E_{0}^{2}=\exp \left\{2\left[\sigma^{2}+\mu+\frac{1}{2} \ln N c+\frac{1}{2} \ln \ln (1 / N c)\right]\right\},
$$

the required distribution may be taken as lognormal with parameters $\sigma^{\prime}=\sigma$, and

$$
\mu^{\prime}=\mu+\frac{1}{2} \ln N c+\frac{1}{2} \ln \ln (1 / N c) .
$$

The distribution of $E$ will therefore be the sum of this lognormally distributed vector with amplitude $E_{0}$ and the Rayleigh-distributed vector $\hat{Z}$.

The required distribution of $E$ is therefore in analogy with (5.6) given by

$$
P(E)=\frac{2 E}{M} e^{-E^{2} / M} \int_{0}^{\infty} p_{0}\left(E_{0}\right) e^{-E_{0}^{2} / M} I_{0}\left(2 U_{0} E / M\right) d E_{0}
$$

where $M=\left\langle Z^{2}\right\rangle$ and $p_{0}\left(E_{0}\right)=\lambda\left(E_{0} ; \sigma, \mu^{\prime}\right)$.

Since, in our case, $E_{0}^{2}$ is much larger (1/Nc times) than $M,(5.15)$ turns into $p_{0}\left(E_{0}\right)$ for large $E_{0}$ and into a Rayleigh distribution for small $E_{0}$. Normalizing with respect to the rootmean-square value of $E$, which from $(5.11)$ is

$$
E_{\mathrm{rms}} \approx \sqrt{N c \ln (1 / N c)} e^{\sigma^{2}+\mu}=\left(E_{0}\right)_{\mathrm{rms}},
$$

these two distributions are therefore

$$
P\left(\frac{E}{E_{\mathrm{rms}}}>R\right)=\frac{1}{2}\left[1-\operatorname{erf}\left(\frac{\ln R+\sigma^{2}}{\sigma \sqrt{2}}\right)\right]
$$

for large $R$, and

$$
P\left(\frac{E}{E_{\mathrm{rms}}}>R\right)=e^{-\frac{R^{2}}{N c}}
$$

for small $R$.

For the intermediate (and all other) values of $R$ we find by normalizing and integrating $(5.15)$ :

$$
P\left(\frac{E}{E_{\mathrm{rms}}}>R\right)=\frac{2}{N c \sigma \sqrt{2 \pi}} \int_{R}^{\infty} d E \int_{0}^{\infty} d E_{0} E E_{0}^{-1} \exp \left[-\frac{E^{2}+E_{0}^{2}}{N c}-\frac{\left(\ln E_{0}+\sigma^{2}\right)^{2}}{2 \sigma^{2}}\right] I_{0}\left(\frac{2 E E_{0}}{N c}\right) .
$$

This is the formal solution of our problem; the practically useful solution is given by the asymptotic distributions (5.17) and (5.18). In comparing our results with the measured distribution (section 6) we have used (5.17) and (5.18). For the intermediate region, which turns out to be comparatively small, we have not gone to the trouble of computing the cumbersome integral (5.19), but have followed the example of Crichlow et al. [1960] in joining the curves of the two asymptotic distributions (5.17) and (5.18) by a circular are (dashed section of curves in figures 3 to 5 ).

\section{Comparison With Experiment and Applications}

Crichlow et al. [1960] have made an empirical analysis of more than 100 amplitudedistribution curves of atmospheric noise measured in Colorado, and further curves measured in Alaska, Panama, Florida, and England. In all these cases they found that when the distribution curve (1.1) is plotted on Rayleigh paper, the measured points lie for small $R$ on a straight line with Rayleigh slope, which is in agreement with (5.18), and on a straight line with larger slope for large $R$; this is again in agreement with (5.17) for as shown in the appendix, the lognormal distribution will for large $R$ plot as a straight line on Rayleigh paper and its slope is determined by the parameter $\sigma$. 
The position of the Rayleigh line is determined by a single point (its slope is already determined by the coordinate net). From (5.18) it follows that for

we obtain $P\left(E / E_{\mathrm{rms}}>R\right)=e^{-1} \approx 37 \% .^{3}$

$$
R=\sqrt{N c}
$$

Thus the parameter $N c$ determines the position of the Rayleigh line, $\sigma$ determines the slope of the high-voltage, low-probability line and the parameters $\sigma, N c$, and $\mu$ determine the rms value of the atmospheric noise $E_{\mathrm{rms}}$. From the above it follows that the distribution (5.17) to (5.19) must always be in agreement with the measured distributions analyzed by Crichlow et al. [1960]. Examples are shown in figures 3, 4, and 5. The distribution agrees equally well with the results measured by Watt and Maxwell [1957].

The distribution derived here is uniquely determined by three parameters: $N c, \sigma$, and $\mu$. The first of these depends only on the properties of atmospheric discharges ( $N$ the number of discharges per unit time, $a$ the time constant of decay); $\sigma$ is the standard deviation of the total attenuation, which in accordance with section 2 is almost exclusively determined by the properties of the propagation path " "almost" because to some small extent it depends on the standard deviation of the logarithm of the peak power of the discharge); $\mu$ is the mean value of the total attenuation and is also predominantly determined by the properties of the propagation path (this parameter drops out in the normalization with respect to the rms value).

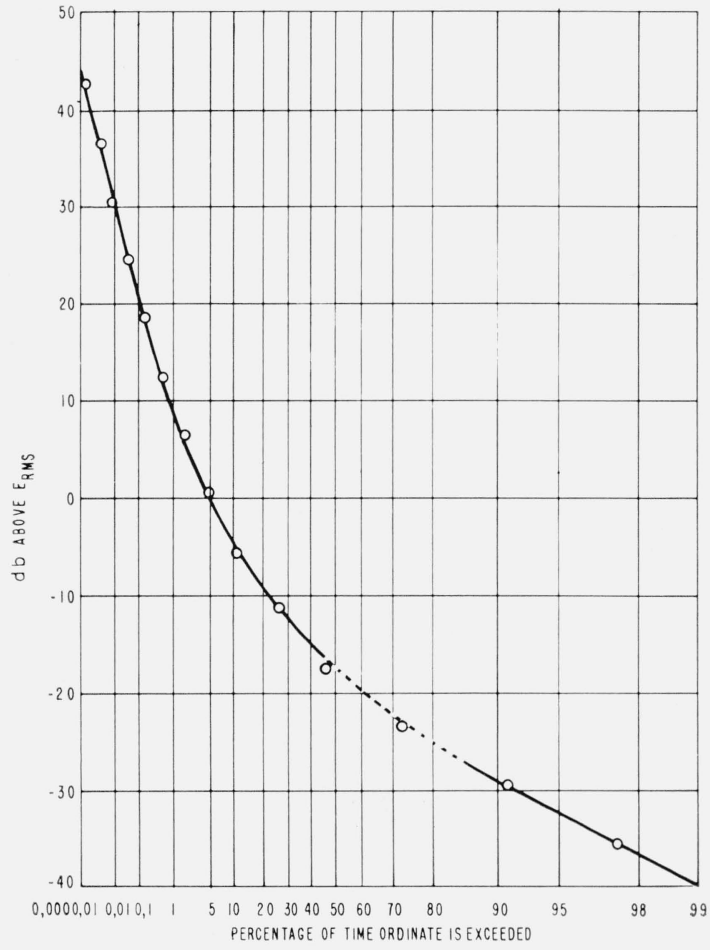

Frgure 3. Amplitude-probability distribution of atmospheric radio noise.

Circles: values measured by Crichlow et al., [1960] on $13.3 \mathrm{kc} / \mathrm{s}$ at Boulder, Colo., October 6, 1958. Full curve: distribution computed from $(5,17)$ and $(5.18)$ for $\sigma=1.67, N c=0.01$. Broken curve interpolated.

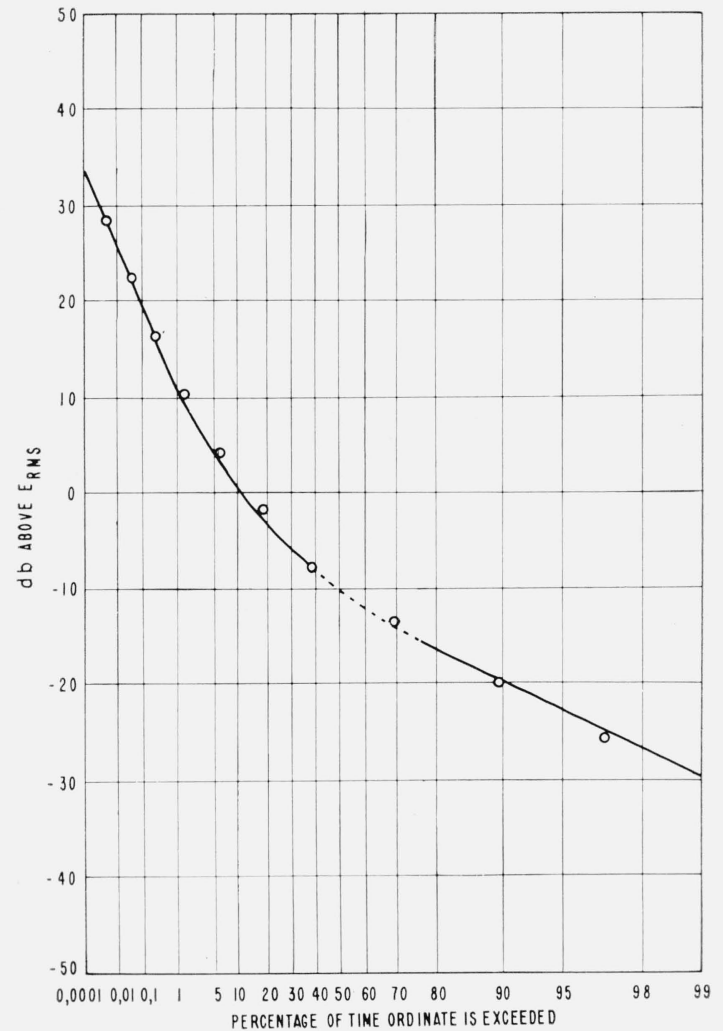

Figure 4. Amplitude-probability distribution of atmospheric radio noise.

Circles: values measured by Crichlow et al., [1960] on $10 \mathrm{Me} / \mathrm{s}$ at Boulder, Colo, September 10, 1958. Full curve: distribution computed from (5.17) and (5.18) for $\sigma=1.15, N c=0.1$. Broken curve interpolated.

3 This point is usually not marked on the $P$ scale; a better policy is therefore to determine the point corresponding to $P=99$ percent, which, as may be verified from (5.18), lies almost exactly $20 \mathrm{db}$ below the rms value of the Rayleigh component (6.1). 


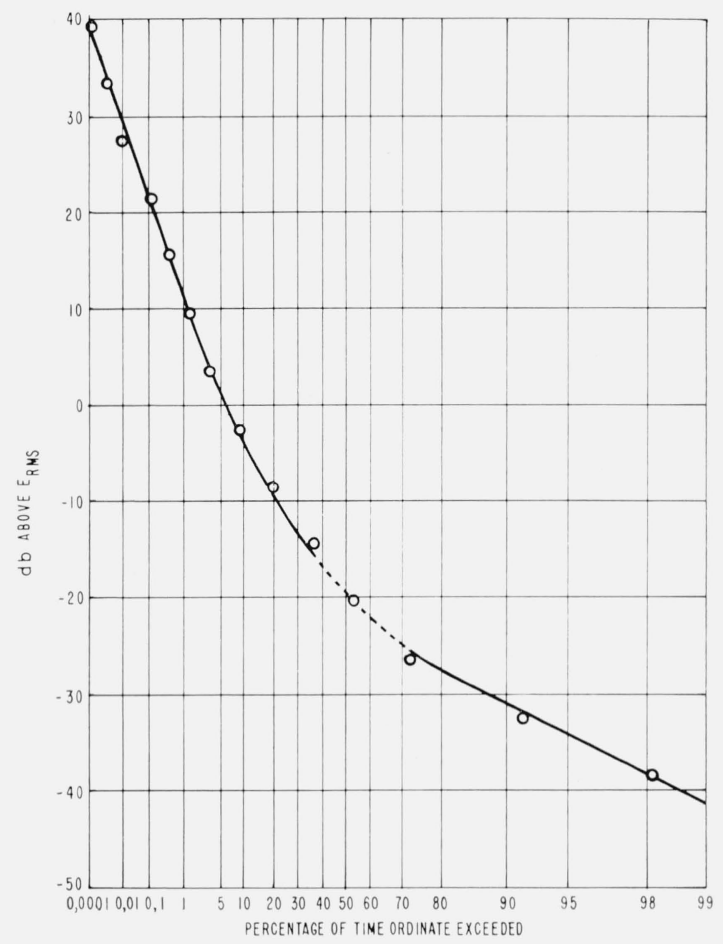

Figure 5. Amplitude-probability distribution of atmospheric radio noise.

Circles: values measured by Crichlow et al., [1960] on $113 \mathrm{kc} / \mathrm{s}$ at Boulder, Colo., September 12, 1958. Full curve: distribution computed from (5.17) and (5.18) for $\sigma=1.74, N_{c}=0.008$. Broken computed interpolated.

Applications to geophysical research are evident: a change of the above parameters will cause a change in the amplitude-distribution curves as measured at different receiving points, times, and under different conditions. Comparison of the measured curves with the theoretical curves should permit the assessment of the causes resulting in the particular measured distribution.

Among other applications of the theoretical distribution, the following one seems hopeful: the variations of the level of atmospheric noise may be caused by a change in propagation conditions or lightning activity or both, and it has been an obstacle to geophysical research that the two causes are difficult to separate. The theoretical distribution derived above should enable the two causes to be separated: if there is an increase in lightning activity, the parameter $N c$ will increase, which will prolong the Rayleigh portion of the curve and raise it with respect to the rms value. If propagation conditions change, this will affect the parameter $\sigma$ and hence the slope of the high-voltage, low-probability portion of the curve.

The problem of the amplitude distribution of radio signals scattered by ionized meteor trails is very similar to the problem treated here, since the occurrence of meteor trails, like that of atmospherics, forms a Poisson process in time and the echo (often) decays exponentially. The method used here may therefore in principle be applied to improve the theory of that distribution [Beckmann, 1962c]. The main difficulty is that the distribution of the peak values (dependent on the distribution of the mass of meteors) as used in astronomy has no rms value.

The method of calculating the overlap of time-dependent (decaying) phenomena whose occurrence is Poisson distributed in time as introduced in section 4 may have applications for queueing and inventory processes. 
The main points of this paper were worked out at the Institute of Radio Engineering and Electronics, Czechoslovak Academy of Sciences. ${ }^{4}$ It was rewritten in a revised form after A. D. Spaulding of the NBS Boulder laboratories had made a thorough study of the original version and pointed out some errors in it; the author wishes to express his sincere gratitude to A. D. Spaulding. The author is also grateful to Mrs. Charlotte Cranford, who typed the manuscript.

\section{Appendix. Mapping of Distribution Functions in Generalized Probability Coordinates}

Let the distribution of a random variable $E$ be given by the relation

$$
P(E>R)=P(R)
$$

We wish to map this distribution in Cartesian $(\xi, \eta)$ coordinates with scales $\xi_{0} \xi(P), \eta_{0} \eta(R)$ chosen in such a way that (A.1) will be mapped in these coordinates as a straight line. The moduls or scaling constants $\xi_{0}, \eta_{0}$ convert $\xi$ and $\eta$ to centimeters, inches, or other units of length and we set them equal to unity.

Since (A.1) is always a nonincreasing function, the design of such coordinates is always possible. One of the two scales, usually $\eta(R)$, may be chosen arbitrarily (e.g., linear, logarithmic, etc.), the other is then determined by the requirement

$$
\eta(R)=C+S \xi(P)
$$

where $C$ and $S$ are choosable constants ; $S$ is the slope of the required straight line.

Let $R(P)$ be the inverse function to $P(R)$ as given by (A.1); then from (A.2) the required scale of the $\xi$ axis is

$$
\xi(P)=\frac{C-\eta[R(P)]}{S}
$$

which is the general solution of the problem.

For the Rayleigh distribution

$$
P(E>R)=\exp \left(-\frac{R^{2}}{E^{2}}\right)
$$

the inverse function is

$$
R(P)=E_{\mathrm{rms}} \sqrt{\ln (1 / P)} .
$$

If we choose

$$
\eta(R)=M \log _{10} R=M^{\prime} \ln R
$$

(where the constants $M$ or $M^{\prime}$ may be absorbed into the modul $\eta_{0}$, so that we may set either equal to unity), we obtain the scale $\xi(P)$ of Rayleigh paper on which (A.4) will be mapped as a straight line with slope $S$, by substituting (A.5) in (A.6) and the result in (A.3):

$$
\xi(P)=\frac{1}{2 S} \log [\ln (1 / P)]+C_{1}
$$

where $C_{1}$ is a constant determining the position of the origin on the $\xi$ axis; we set it equal to zero $^{5}$. The slope $S$ for Rayleigh paper is usually chosen as $S=-1$ or $S=-1 / 2$. The latter is used in figures 1 and 3 to 5 of this paper.

4 URE-ČSAV Inst. Rept. No. 26, 1962.

${ }^{5}$ Since either $M$ or $M^{\prime}$ in (A.6) may be set equal to unity, the symbol " $\log$ " in (A.7) may mean either $\log _{10}$ or $\ln$. 
It may be shown by elementary methods that all functions of the kind

$$
P(R)=\exp \left(-k R^{m}\right)
$$

and only these functions will map on Rayleigh paper (A.6), (A.7) as straight lines. The slope of such a line is

$$
S^{\prime}=\frac{2 S}{m}
$$

The curve that will result from mapping a general function $P(R)$ on Rayleigh paper is found by substituting $P(R)$ in (A.7) and eliminating $R$ from (A.7) and (A.6), so that the curve $\eta=\eta(\xi)$ will be obtained. For example, for the lognormal distribution normalized as in $(2.9)$ we have

$$
P(R)=\frac{1}{2} \operatorname{erfc} x
$$

where

$$
\operatorname{erfc} x=1-\operatorname{erf} x
$$

and

$$
x=\frac{\ln R+\sigma^{2}}{\sigma \sqrt{2}}=\frac{\eta+\sigma^{2}}{\sigma \sqrt{2}} .
$$

In the second expression of (A.12) we have substituted from (A.6) for $M^{\prime}=1$. The relation between $\xi$ and $\eta$ is therefore

$$
\xi=\frac{1}{2 S} \ln \left[\ln 2-\ln \operatorname{erfc}\left(\frac{\eta+\sigma^{2}}{\sigma \sqrt{2}}\right)\right]
$$

This (or any other) function will be mapped on Rayleigh paper as a straight line in those regions where $d \xi / d \eta=$ const. From (A.12) and (A.13)

$$
\frac{d \xi}{d \eta}=\frac{d \xi}{d x} \frac{d x}{d \eta}=\frac{e^{-x^{2}}}{S \sigma \sqrt{2 \pi}(\ln 2-\operatorname{erfc} x) \operatorname{erfc} x}
$$

This expression will be constant for $x$ constant, i.e., from (A.12) for

$$
|\ln R|<<\sigma^{2}
$$

making $x \approx \sigma / \sqrt{2}$, which substituted in (A.14) yields $1 / S^{\prime}$, where $S^{\prime}$ is the slope of this straight line. The ratio of $S^{\prime}$ to $S$ (where $S$ is the slope for which the Rayleigh paper was designed), is therefore

$$
\frac{S^{\prime}}{\mid S}=\left(S \frac{d \xi}{d \eta}\right)^{-\perp}=\sigma \sqrt{2 \pi} e^{\sigma^{2} / 2}\left[\ln 2-\ln \operatorname{erfc} \frac{\lceil\sigma}{\sqrt{2}}\right] \operatorname{erfc} \frac{\sigma}{\sqrt{2}} .
$$

Thus the lognormal distribution will map on Rayleigh paper approximately as a straight line in the region where (A.15) holds, i.e., in the region of small probabilities for $\sigma^{2}>1$ (cf, fig. 1, p. 724).

One way of determining $\sigma$ from a measured amplitude-distribution of atmospheric noise is to plot the upper portion of the curve on Gaussian probability paper with the $R$ axis given in decibels. If this part of the curve is lognormal, the points will lie on a straight line and $\sigma^{\prime}$ may be determined, e.g., as the decibel difference between the 2.28 percent and 0.135 percent levels. The parameter $\sigma$ is then found from the relation

$$
\sigma^{\prime}=20 \sigma \log _{10} e=8.686 \sigma .
$$




\section{References}

Beckmann, P. (1962a), Deviations from the Rayleigh distribution for a small and for a random number of interfering waves, Práce URE-ČSAV No. 25.

Beckmann, P. (1962b), The probability distribution of random vector plus a Rayleigh-distributed vector and its applications, Práce URE-ČSAV No. 23.

Beckmann, P. (1962c), The amplitude distribution of radio waves scattered by meteor trails. Bull. Astr. Cz. 13, 232-236.

Blanc-Lapierre, A., R. Fortet (1953), Théorie des fonctions aleatoires, Masson, Paris.

Crichlow, W. Q., C. J. Roubique, A. D. Spaulding, W. M. Beery (1960), Determination of the amplitudeprobability distribution of atmospheric radio noise from statistical moments, J. Res NBS 64D (Radio Prop.), No. 1, 49-56.

Foldes, G. (1960), The lognormal distribution and its applications to atmospheric studies. Statistical Methods in Radio Wave Propagation, ed. W. C. Hoffman, pp. 227-232 (Pergamon Press, London-New York, ParisLos Angeles).

Furutsu, K., and T. Ishida (1961), On the theory of amplitude distribution of impulsive random noise, J. Appl. Phys. 32, 1206-1221.

Gnedenko, B. V. (1962), The theory of probability (Chelsea Publishing Co., New York, N.Y.).

Horner, F., and J. Harwood (1956), An investigation of atmospheric noise at very low frequencies, Proc. IEE 103B, $743-751$.

Watt, A. D., and E. L. Maxwell (1957), Characteristics of atmospheric noise on 1-100 kc/s, Proc. IRE 45, $787-794$.

Wheelon, A. D. (1962), Amplitude distribution for radio signals reflected by meteor trails, II, J. Res. NBS 64D (Radio Prop.) 241-247.

(Paper 68D6-370) 